\title{
Multiple Pyoderma Gangrenosum Ulcers Associated with Cocaine Abuse
}

"Ricardo Ruiz-Villaverde ${ }^{1}$ and Daniel Sánchez-Cano ${ }^{2}$
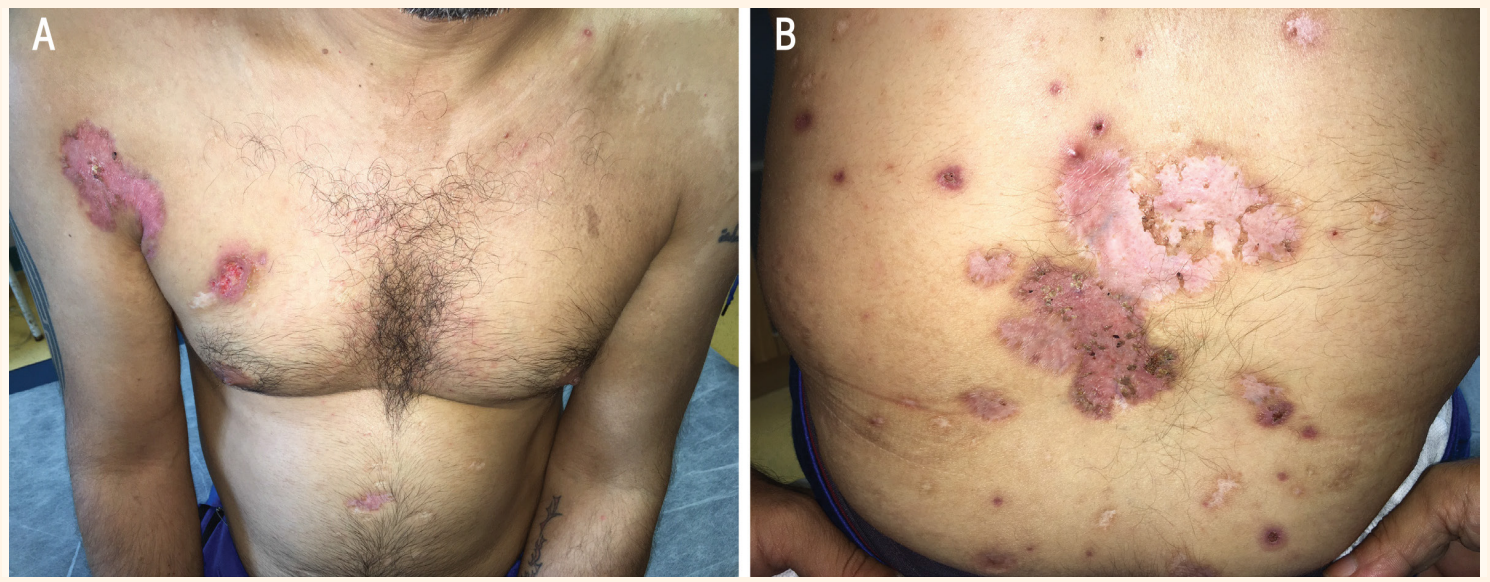

Figure 1: A: Erythematous-violaceous ulcerated plaques on the right armpit and chest of a 38-year-old male cocaine user. B: Erythematous plaques with serous crusts on the back of the patient, with small disseminated ulcers surrounding the major lesions.

\section{A}

38-YEAR-OLD MALE WAS ADMITTED TO THE Department of Dermatology, Complejo Hospitalario de Granada, Granada, Spain, in 2016 with inflamed papules, plaques and nodules in the armpits, chest, pubis and lumbar region [Figure 1]. He had a history of poorly-controlled type 2 diabetes, which was being treated with insulin, and dyslipidaemia, treated with $40 \mathrm{mg}$ of simvastatin per day. The skin lesions were associated with abscess formation and spontaneous drainage. The patient acknowledged a past history of cocaine abuse, but denied any recent drug use. The results of a laboratory work-up were normal, including a complete blood count, general biochemistry tests, an autoimmune profile, an antinuclear antibody panel, a lupus anticoagulant and cryoglobulin work-up, a C-reactive protein test, complement levels and erythrocyte sedimentation rate. The patient tested negative for hepatitis $B$, hepatitis $C$ and human immunodeficiency virus infections. A computed tomography scan showed neither pulmonary nor abdominal anomalies. Cutan- eous tissue cultures for bacteria, fungi and mycobacteria were all negative. The initial differential diagnosis included cutaneous chronic lupus and atypical mycobacteriosis.

An excisional biopsy was performed on an active lesion on the right side of the chest. Histopathology revealed a dense neutrophilic inflammatory infiltrate involving the entire dermis, without granulomas or vasculitis [Figure 2]. Direct immunofluorescence testing was negative. Urine toxicology screening was positive for cocaine and levamisole. As a result of the clinical and pathological findings, a diagnosis of pyoderma gangrenosum (PG) was made. The patient was advised to stop using cocaine and was prescribed prednisone at a dose of $60 \mathrm{mg}$ daily for a month, reduced by $5 \mathrm{mg}$ per week until cessation. Unfortunately, due to patient noncompliance, optimal control of the dermatological condition could not be achieved. Continued use of cocaine resulted in ongoing PG flare-ups. The patient was subsequently prescribed $0.5 \mathrm{mg} / \mathrm{kg} /$ day of prednisone, although 


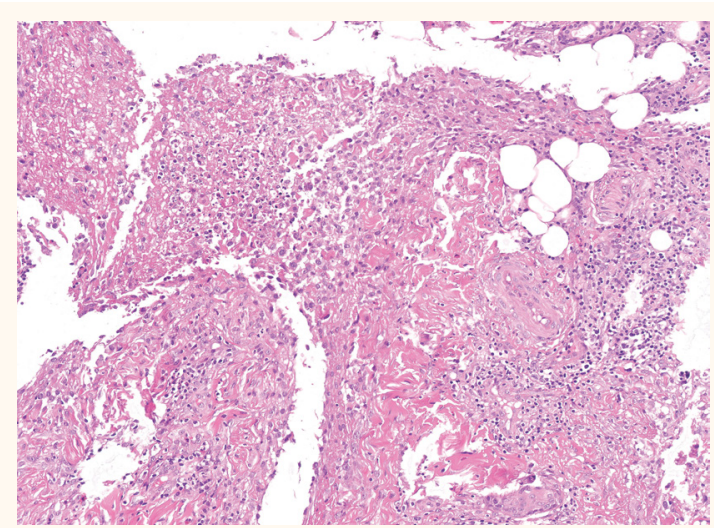

Figure 2: Haematoxylin and eosin stain at $\mathrm{x} 40$ magnification showing a dense neutrophilic inflammatory infiltrate involving the entire dermis, without evidence of granulomas or vasculitis.

he never completed treatment cycles for longer than three weeks. After one year, the patient was lost to follow-up.

\section{Comment}

PG is an uncommon neutrophilic dermatosis that presents as an inflammatory and ulcerative disorder of the skin. Most cases are associated with an underlying immunological condition, such as inflammatory bowel disease, rheumatological diseases, haematological disorders or metabolic syndrome. ${ }^{1}$ In the current patient, all of the laboratory tests were negative apart from the toxicology screening, which distinguishes this case from others reported in the literature. The differential diagnosis included connective tissue diseases such as chronic cutaneous lupus and granulomatosis with polyangiitis. Infectious diseases such as atypical mycobacteriosis, an emerging disease in Spain, were also considered. ${ }^{2}$

Keith et al. previously reported an association between PG and the use of levamisole-adulterated cocaine. ${ }^{3}$ Endothelial damage due to the direct toxic effect of cocaine and vasculopathy caused by levamisole have been postulated to be the major pathophysiological mechanisms in these types of cases. ${ }^{4}$ The therapeutical approach may be complicated; the severity of PG influences the choice of the initial therapy. Systemic therapy-such as corticosteroids, immunosuppressive therapy or even biological therapy-is needed to achieve remission in cases of extensive PG; cessation of cocaine use is also imperative. ${ }^{5}$

\section{References}

1. Roche E, Martínez-Menchón T, Sánchez-Carazo JL, Oliver V, Alegre de Miquel V. [Two cases of eruptive pyoderma gangrenosum associated with cocaine use]. Actas Dermosifiliogr 2008; 99:727-30. doi: 10.1016/S1578-2190(08)70351-2.

2. Esteban J, Ortiz-Pérez A. Current treatment of atypical mycobacteriosis. Expert Opin Pharmacother 2009; 10:2787-99. doi: $10.1517 / 14656560903369363$.

3. Keith PJ, Joyce JC, Wilson BD. Pyoderma gangrenosum: A possible cutaneous complication of levamisole-tainted cocaine abuse. Int J Dermatol 2015; 54:1075-7. doi: 10.1111/ijd.12212.

4. Baliu-Piqué C, Mascaró JM Jr. Multifocal and refractory pyoderma gangrenosum: Possible role of cocaine abuse. Australas J Dermatol 2016. doi: 10.1111/ajd.12498.

5. Jeong HS, Layher H, Cao L, Vandergriff T, Dominguez AR. Pyoderma gangrenosum (PG) associated with levamisoleadulterated cocaine: Clinical, serologic, and histopathologic findings in a cohort of patients. J Am Acad Dermatol 2016; 74:892-8. doi: 10.1016/j.jaad.2015.11.040. 